\title{
Improved centerline tracking for new descriptors of atherosclerotic aortas
}

\author{
Hugo Gangloff \\ ICube, Université de Strasbourg \\ CNRS UMR 7357 \\ Illkirch, FRANCE \\ GEPROVAS \\ Strasbourg, FRANCE \\ hugogangloff@unistra.fr \\ Mickaël Ohana \\ ICube, Université de Strasbourg \\ CNRS UMR 7357 \\ Illkirch, FRANCE \\ Department of Radiology, CHRU \\ Strasbourg, FRANCE \\ mickael.ohana@chru-strasbourg.fr
}

\author{
Emmanuel Monfrini \\ SAMOVAR \\ Télécom SudParis \\ Institut Polytechnique de Paris \\ Évry, FRANCE \\ emmanuel.monfrini@telecom-sudparis.eu \\ Christophe Collet \\ ICube, Université de Strasbourg \\ CNRS UMR 7357 \\ Illkirch, FRANCE \\ c.collet@unistra.fr
}

\author{
Mohamed Zied Ghariani \\ GEPROVAS \\ Strasbourg, FRANCE \\ Department of Vascular Surgery \\ and Kidney Transplantation, CHRU \\ Strasbourg, FRANCE
}

mohamed-zied.ghariani@chru-strasbourg.fr

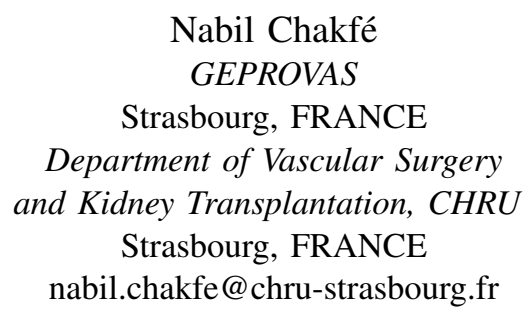

Nabil Chakfé

GEPROVAS

rg, FRANCE

Department of Vascular Surgery

Strasbourg, FRANCE

nabil.chakfe@chru-strasbourg.fr

\begin{abstract}
To develop more patient specific treatments we need to collect and understand a growing set of data about the patient. In the context of the atherosclerotic aortic bifurcation, following recent developments in clinical research, we propose new objective mathematical descriptors of the patient vascular morphology. These descriptors can be automatically extracted thanks to a robust centerline extraction algorithm also presented in this article and that is dedicated to the processing of noisy meshes. We show that these descriptors can provide new vizualization tools built from segmented CT scans.

Index Terms-image processing and analysis, centerline extraction, data visualization, patient specific medecine
\end{abstract}

\section{INTRODUCTION}

Vascular diseases are a major cause of death in the world and these pathologies are not completely understood yet. The efforts for developping patient specific treatments are crucial to improve the clinical outcomes and our comprehension of the diseases. In the context of the atherosclerotic aortic bifurcation (see Figure 1 for a X-ray scan depicting the anatomy), the physician planning a surgical procedure would greatly benefit from more precise and objective data to gain insight about each patient's specific case [7] [9].

In this paper we first propose an original and automatic approach for the centerline extraction in noisy 3D meshes. It is then applied to compute automatically objective mathematical descriptors over the patient's aortic morphology. Such

The authors would like to thank association GEPROVAS for the funding of this work (https://geprovas.org).

978-1-7281-8750-1/20/\$31.00 @2020 IEEE

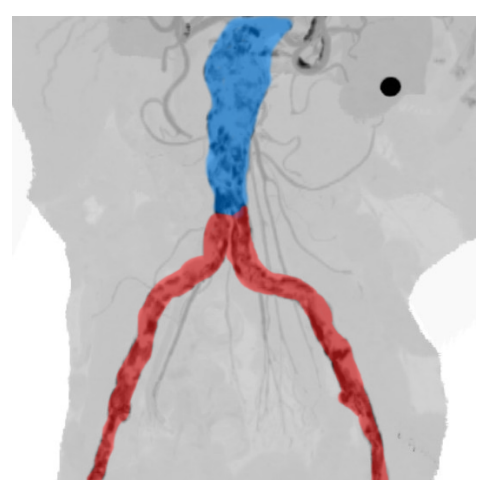

Fig. 1: CT-scan reconstruction of an atherosclerotic aortic bifurcation. The colored parts denote the three segments we will consider: in blue, the terminal aortic section (starting from the inferior mesenteric artery), in red, the iliac arteries.

information is extracted from a patient's segmented CT scan. We present these descriptors and their computation.

These descriptors constitute enhanced data that physicians can use for a more patient specific approach to the treatment (choice of the vascular procedure, sizing of the biomedical device to implant, knowledge of the patient morphology...). The descriptors can also be used for a more fundamental research work to develop a better understanding of the atherosclerotic disease. Indeed, the recent progress in medical imaging and segmentation tools now enable clinical research to go one step further and exploit more precisely new type of data. In the context of atherosclerotic pathologies, recent articles develop metrics and descriptors to describe the calcificied areas [2] [6]. 


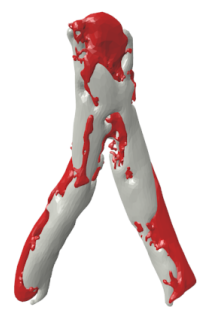

(a)

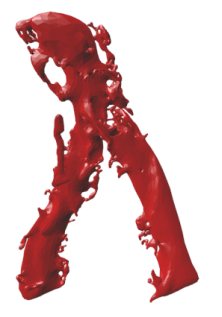

(b)

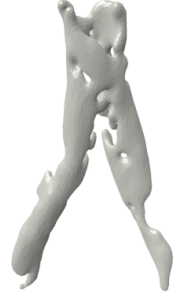

(c)
Fig. 2: An example of the two meshes used as input data: in red the aorta lumen and in gray the calcifications. Fig. 2a: the meshes are shown in their original configuration. Fig. $2 b$ and Fig. 2c: individual meshes.

For our study, CT scans of the atherosclerotic aortic bifurcation of 55 patients were collected. Precisely, as depicted in Figure 1, we consider the terminal aorta as well as the two iliac arteries. The aortic lumen meshes as well as the calcification meshes were manually segmented by a radiologist. The two 3D objects obtained, illustrated in Figure 2, are the input data of our approach for centerline tracking and to build quantifiers to objectively describe the patient morphologies.

\section{CEnTERline TRACKING IN NOISY AORTA MESHES}

The centerline estimation of the aortic lumen is a crucial step in many medical applications, including the present one, in which we need an estimation of the centerline to compute the calcification descriptors. The aorta meshes we have to deal with are particularly noisy due to the development of atherosclerosis at advanced stages. Even a manual segmentation performed by a radiologist will result in a noisy and corrupted mesh ${ }^{1}$ (see, for example, the red mesh in Figure 2). Indeed, in these pathologic cases, the arterial wall is covered with calcified plaques which alter its structure. The latter being sometimes very noisy even in the case of manual segmentation.

Based on the concept of accumulation map, [4] proposes the ImAcc algorithm to retrieve the centerline of partially sampled meshes. However this algorithm alone fails on most of our cases and we develop a more robust approach to handle the corrupted meshes of our database. Our algorithmic solution first builds a noise-reduced aorta mesh, then computes its accumulation map and finally estimate the centerline via the Minimal Cost Path algorithm. The proposed solution is fully automatic except for the centerline extremities which need to be provided by the user.

The noise-reduced aorta mesh is approximated by using the 3D alpha shape algorithm [1] and then deleting all the possibly remaining inner vertices of the mesh. This is done automatically with the help of the Blender ${ }^{2}$ software. We refer to this step as the alpha shape preprocessing step. This enables us to treat very noisy or partially sampled aorta meshes thanks to the envelop constructed with the alpha shape algorithm.

\footnotetext{
${ }^{1}$ The final mesh corruption is also partly due to limitations inherent to the radiologist's software used for segmentation.

${ }^{2}$ http://www.blender.org
}

These meshes augmented with their alpha shapes are then discretized to be used as input for the ImAcc algorithm [4] which produces an output under the form of a probability map where the highest values are the most likely to be near the centerline. In Section IV we show that using our preprocessing step based on the alpha shape improves the quality of the accumulation map produced and thus the estimated centerline. Note that, following ImAcc, [3] proposes to use a second algorithm based on a confidence map which failed in the context of our study. The latter will then be discarded from the comparisons.

Eventually, to form the centerline based on the accumulation map we propose an association between the latter with the Minimal Cost Path (MCP) algorithm. In the result section, we show that such an association is robust for tracking centerlines in very noisy meshes. MCP has already been used for centerline tracking in CT data [5] [8]. Between the two centerline extremities entered by the user, the MCP algorithm will compute an estimation of the aortic lumen centerline through the accumulation map. Using the accumulation map as entry data the MCP is one of the novelties we introduce. The cost function of a voxel from the accumulation map is chosen to be $C(x)=2^{-\operatorname{ImAcc}(x)}$ where $\operatorname{ImAcc}(x)$ refers to the value of the voxel $x$ in the accumulation map. A complete pseudo-code of the MCP algorithm can be found in [5].

The full workflow that we propose is described in Figure 3.

\section{CALCIFICATIONS DESCRIPTORS}

In this section we detail the three descriptors of the calcifications that we have developped in association with clinicians. They are based on recent clinical research in this area [2] [6]. These descriptors also require a finely computed aorta centerline, that is why we presented the algorithm described in Section II. Each of these descriptors will be computed on each of the three segments of the aorta bifurcation (the segments are specified in Figure 1).

\section{A. Distance to centerline (or protrusion)}

We want to evaluate the distance between the calcification mesh and the centerline that we have estimated in the previous section. Such information represent the protrusion of the calcifications over the lumen. Each vertice of the calcification mesh is attributed the value of the Euclidean distance of the nearest point of the centerline.

\section{B. Circumferential repartition rate}

The circumferential repartition of the calcifications along the arterial wall is computed at each point of the centerline. It represents the proportion of the arterial wall covered by calcifications. The technique of ray casting [11] is used, then elementary geometry is performed to detect collisions between each ray and the faces of the calcification mesh.

\section{Saliency}

The saliency, computed with the Gauss-Bonnet curvature [10], is the indicator of the local sharpness of the calcification. It is computed at each vertice of the mesh. 


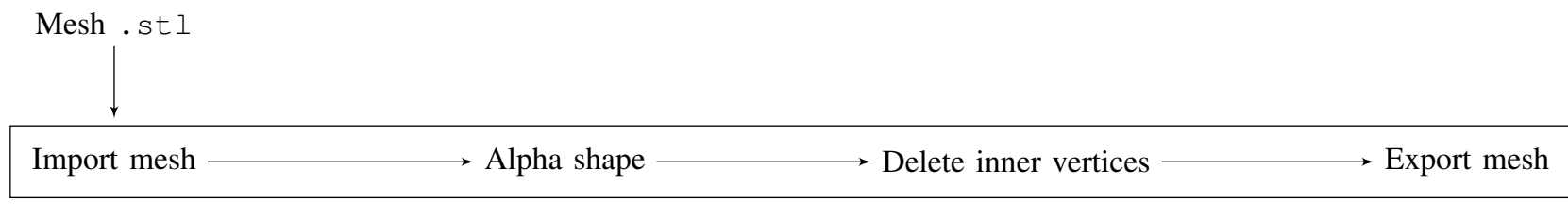

Preprocessing: denoised envelop construction

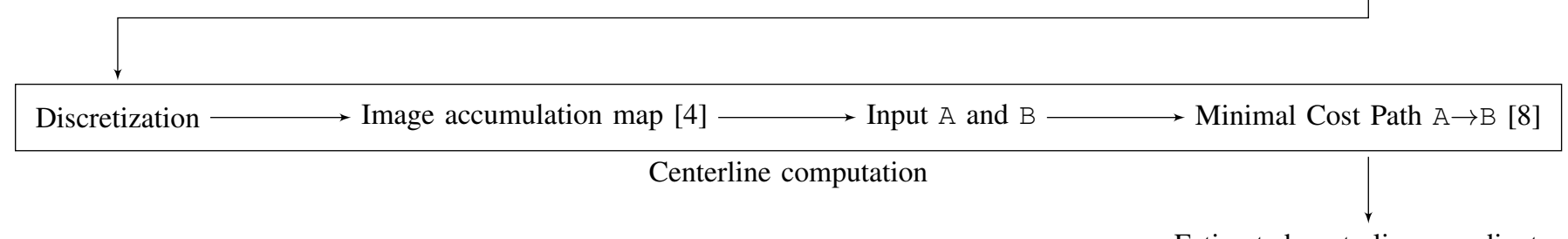

Estimated centerline coordinates

Fig. 3: Workflow of our algorithm for the computation of the centerline on a segment A-B.

\section{EXPERIMENTS AND RESULTS}

We now apply the methodology described above to a cohort of 55 patients that underwent a CT scan of the aortic bifurcation.

\section{A. Improving the estimated centerline}

The first steps consist in the centerline estimation in the noisy mesh of the aorta based on the preprocessing with alpha shape, the ImAcc algorithm and the MCP algorithm.

Figure 4 first illustrates the computation of the 3D alpha shapes on some of the morphologies in the database. Then, in Figure 5, we illustrate the estimated centerlines, using the association of the algorithms ImAcc and MCP using either the preprocessing step (alpha shape computation) or no preprocessing step. The estimated centerlines are overlaying the normalized median values of the accumulation map computed on a projection along the $\mathrm{Y}$ axis. The projected accumulation maps in the left column are much less affected by local clusters of high values that decrease the precision of the MCP algorithm. This leads to better approximated centerlines. Note that the lack of ground truths for such meshes requires us to visually assess the centerline quality. Finally, for a better visualization of these centerlines we provide, in Figure 6, the two estimated centerlines on the same silhouette of the lumen of the aorta projected along each axis. This comforts us in the robutness of the method.

Figure 7 illustrates the estimated centerline on three morphologies from the database. We found out that the method performed well, including on some of the most noisy meshes.

\section{B. Visualization of the descriptors}

Figure 8a depicts some calcification meshes enhanced with the information of the distance to the centerline. Figure $8 \mathrm{~b}$ gives an illustration of the calcification mesh with added saliency information. Figure $8 \mathrm{c}$ illustrates the calcifications circumferential repartition rate along a segment. We note that the centerline estimation is a crucial step for a both the protrusion and the circumferential rate descriptors.

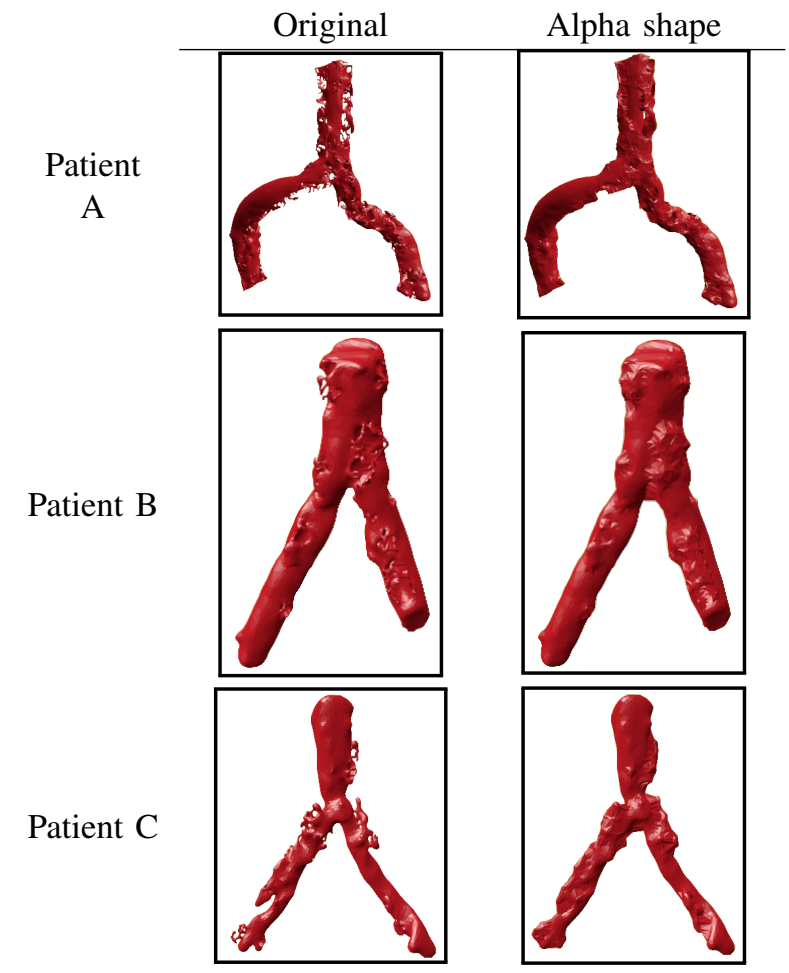

Fig. 4: Illustration of the computation of the $3 \mathrm{D}$ alpha shape of noisy lumen meshes.

\section{CONCLUSION}

In this article we presented a workflow for the estimation of centerlines of noisy meshes. We built a robust approach able to deal with very corrupted data. This development finds a direct application for the centerline estimation of blood vessels affected by calcifications, whose segmentation, even manually, produces degraded meshes. Indeed, the complexity of the data required a novel and dedicated algorithm. The ability to estimate the centerline in such data enabled us to construct new mathematical descriptors of the data, which 


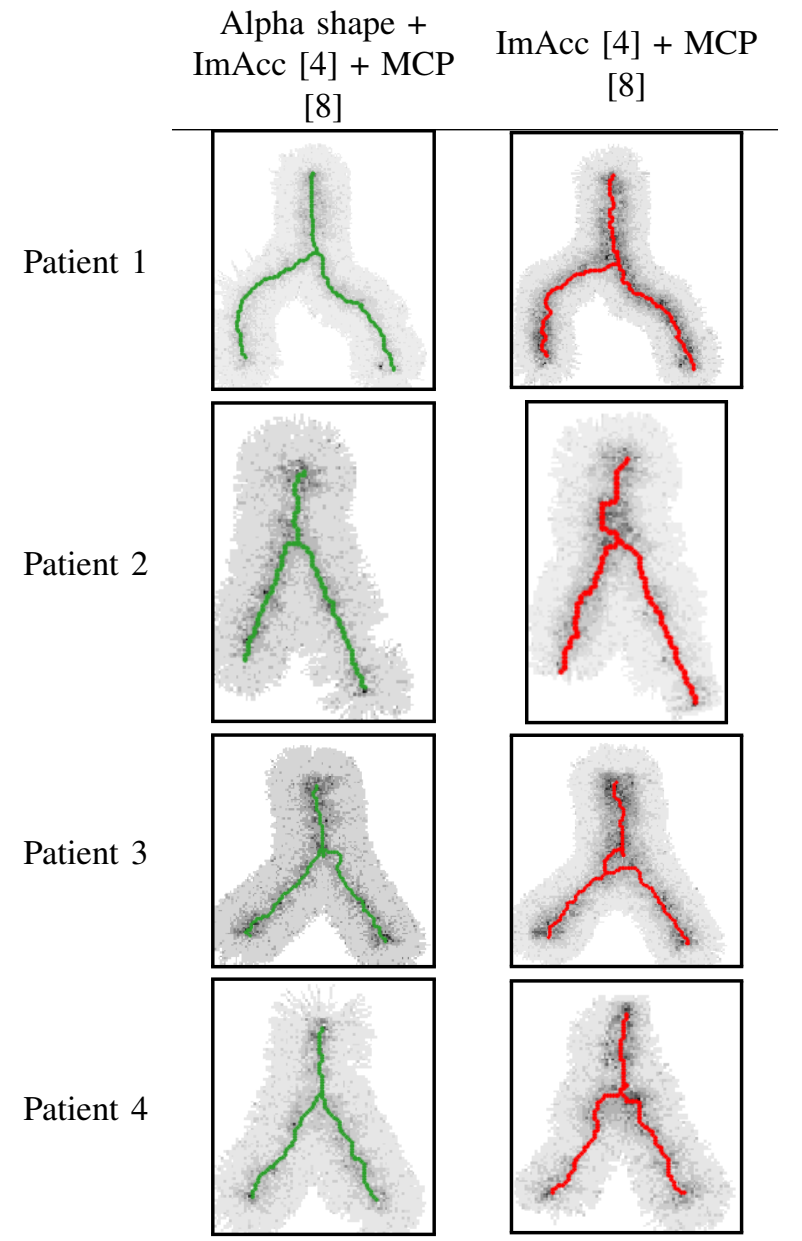

Fig. 5: Estimated centerlines with a prior alpha shape computation (in green) and without a prior alpha shape computation (in red). The centerlines are overlaying the normalized median values of the accumulation map computed on a projection along the $\mathrm{Y}$ axis. The patients correspond to the patients of Figure 6.

form an enhanced visualization of the medical data to better guide clinicians.

The next step in this work will be to correlate the proposed descriptors with clinical outcomes using a machine learning approach. This would in fact be the foundation of an automatic and objective classification of the atherosclerotic aortic bifurcations.

\section{REFERENCES}

[1] Herbert Edelsbrunner and Ernst P Mücke. "Threedimensional alpha shapes". In: ACM Transactions on Graphics (TOG) 13.1 (1994), pp. 43-72.

[2] Peter M Graffy et al. "Automated segmentation and quantification of aortic calcification at abdominal CT: application of a deep learning-based algorithm to a longitudinal screening cohort". In: Abdominal Radiology 44.8 (2019), pp. 2921-2928.
[3] Bertrand Kerautret and al. "Centerline detection on partial mesh scans by confidence vote in accumulation map". In: Pattern Recognition (ICPR), 2016 23rd International Conference on. IEEE. 2016, pp. 1376-1381.

[4] Bertrand Kerautret et al. "3D geometric analysis of tubular objects based on surface normal accumulation". In: International Conference on Image Analysis and Processing. Springer. 2015, pp. 319-331.

[5] Almar Klein and al. "Automatic segmentation of the wire frame of stent grafts from CT data". In: Medical image analysis 16.1 (2012), pp. 127-139.

[6] Sila Kurugol et al. "Automated quantitative 3D analysis of aorta size, morphology, and mural calcification distributions". In: Medical physics 42.9 (2015), pp. 54675478.

[7] Anne Lejay and al. "Arterial Occlusion Is Not Just About Length: There's More than Meets the Eye!" In: European Journal of Vascular and Endovascular Surgery 58 (Feb. 2019).

[8] C Metz and al. "Two point minimum cost path approach for CTA coronary centerline extraction". In: The Insight Journal 12 (2008).

[9] Mickaël Ohana and al. "Detailed cross-sectional study of 60 superficial femoral artery occlusions: morphological quantitative analysis can lead to a new classification". In: Cardiovascular diagnosis and therapy 4.2 (2014), p. 71.

[10] Sreenivas Sukumar and al. "Shape measure for identifying perceptually informative parts of 3D objects". In: Third International Symposium on 3D Data Processing, Visualization, and Transmission (3DPVT'06). IEEE. 2006, pp. 679-686.

[11] Onno Wink and al. "Fast delineation and visualization of vessels in 3-D angiographic images". In: IEEE transactions on medical imaging 19.4 (2000). 


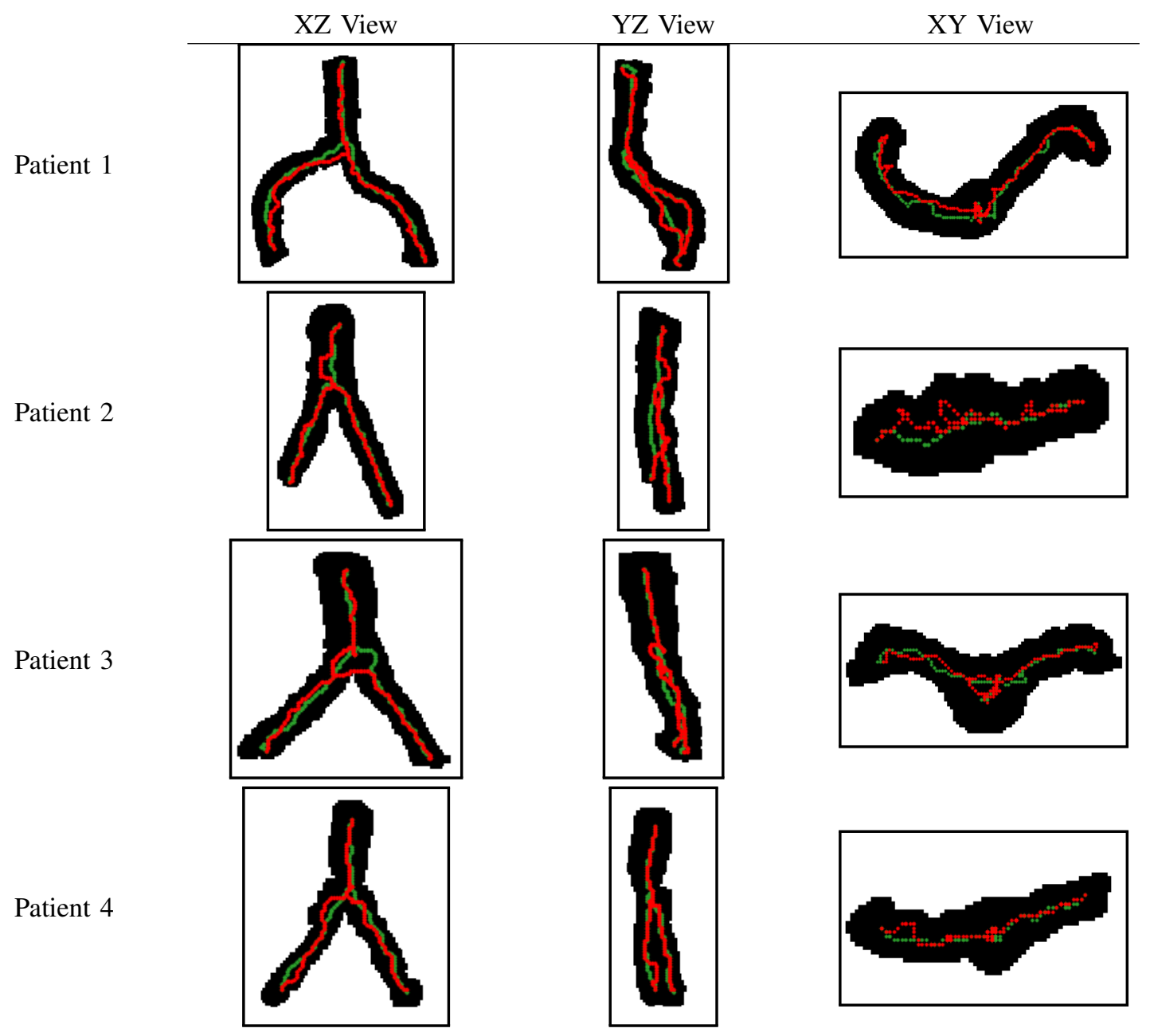

Fig. 6: In green the centerline with the alpha shape preprocessing and in red the centerline without the preprocessing overlaying the projections of the lumen of the aortic bifurcation along each axis. The patients correspond to the patients of Figure 5 .
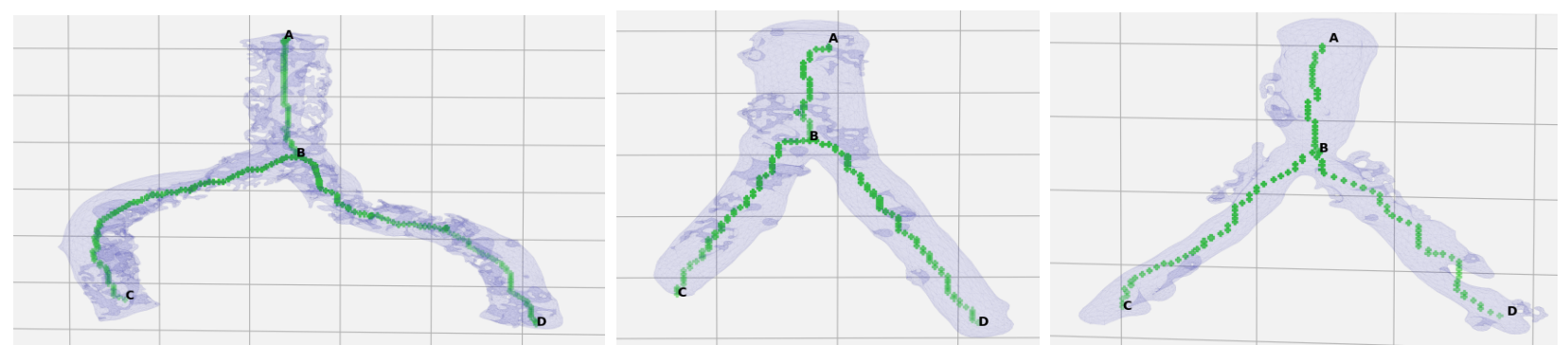

Fig. 7: Some estimated centerlines (in green) resulting from our method, over the three segments (3D views). The terminal aorta mesh appears in blue. One can note the noisy surface which requires a robust estimation technique as we have developped. 

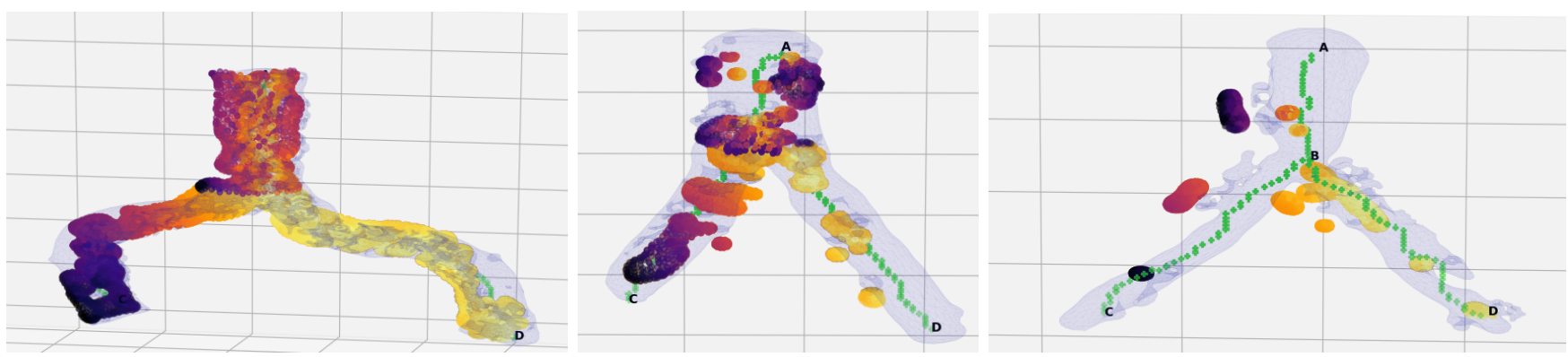

(a) Protrusion: the color of the vertices of the calcification mesh represents the distance to the centerline of that vertice. The darker the color the farther the vertice.

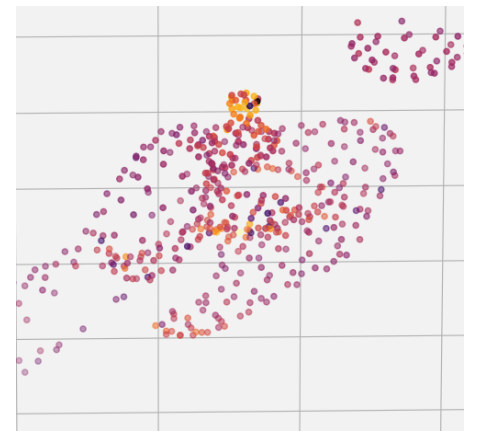

(b) Saliency: the color of the vertices of the calcification mesh represents the local curvature. A darker color indicates a bigger curvature.

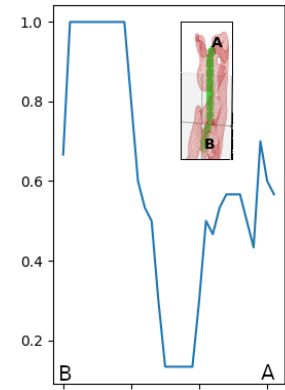

(c) Circumferential rate: Rate of the arterial wall (in red in the miniature) covered by the calcification, along the segment BA.

Fig. 8: Illustrations of the calcification descriptors (3D views). 